\title{
Electrons, pseudoparticles, and quasiparticles
} in one-dimensional interacting electronic systems

\author{
J. M. P. Carmelo ${ }^{1}$, A. H. Castro Neto ${ }^{2}$, and N. M. R. Peres ${ }^{1}$ \\ ${ }^{1}$ Department of Physics, University of Évora, Apartado 94, P-7001 Évora Codex, Portugal \\ ${ }^{2}$ Institute of Theoretical Physics, University of California, Santa Barbara, CA 93106-4030
}

(Received 24 February 1995)

\begin{abstract}
We find the singular transformation between the electron operator and the pseudoparticle operators for the Hubbard chain. We generalize the concept of quasiparticle to one-dimensional electronic systems which in 1D refers to many-pseudoparticle objects. We obtain explicit results for the electron renormalization factor, self energy, and vertex functions at the Fermi points. We also discuss the possible connection of our results to higher dimensions and explore the possibilities of instabilities in the interacting problem such as the formation of Cooper pairs.

PACS numbers: 72.15. Nj, 74.20. -z, 75.10.Lp, 67.40. Db
\end{abstract}

Typeset Using REVTEX 
The unconventional electronic properties of novel materials such as the superconducting coper oxides and synthetic quasi-unidimensional conductors has attracted much attention to the many-electron problem in spatial dimensions $1 \leq \mathrm{D} \leq 3$. Although quantum liquids in dimensions $1<\mathrm{D}<3$ are, probably, neither Fermi liquids (3D) nor Luttinger liquids (1D) but have instead an intermediate physics, the complexity of the problem requires a good understanding of both the different and common properties of these two limiting cases. While their different properties were the motivation for the introduction of the concept of Luttinger liquid in 1D [1], the characterization of their common properties is also of great interest because the latter are expected to be present in dimensions $1<\mathrm{D}<3$ as well. One example is the Landau-liquid character common to Fermi liquids and some Luttinger liquids which consists in the generation of the low-energy excitations in terms of different momentum-occupation configurations of quantum objects (quasiparticles or pseudoparticles) whose forward-scattering interactions determine the low-energy properties of the quantum liquid. This generalized Landau-liquid theory was introduced in Ref. [2], which refers to contact-interaction soluble problems (shortly after the same kind of ideas were applied to $1 / r^{2}$-interaction integrable models [3]).

The nature of interacting electronic quantum liquids in dimensions $1<\mathrm{D}<3$, including the existence or non existence of quasiparticles and Fermi surfaces, remains an open question of crucial importance for the clarification of the microscopic mechanisms behind the unconventional properties of the novel materials. Inspired by the results of Landau's theory of the Fermi liquid in 3D, it is one of the aims of this Letter to introduce the operator which creates a well defined elementary excitation of the Fermi system, which we call quasiparticle. This excitation is a transition between two exact ground states of the interacting electronic problem differing in the number of electrons by one. When one electron is added to the electronic system the number of these excitations also increases by one. Naturally, its relation to the electron will depend on the overlap between the states associated with this and the quasiparticle and how close we are in energy from the starting interacting ground state. Therefore, in order to define the quasiparticle we need to understand the properties of the 
actual ground state of the problem as, for instance, is given by its exact solution via the Bethe ansatz (BA). The vanishing of the one-electron renormalization factor in $1 \mathrm{D}$ does not necessarily implies the non existence of the above quasiparticles and clarifying the problem in the 1D limit is also important for understanding how dimensionality changes the physics of interacting electronic systems in dimensions $1 \leq \mathrm{D} \leq 3$.

We consider here the Hubbard model 44 in one dimension with a finite chemical potential $\mu$ and in the presence of a magnetic field $H$,

$\hat{H}=-t \sum_{j, \sigma}\left[c_{j, \sigma}^{\dagger} c_{j+1, \sigma}+c_{j+1, \sigma}^{\dagger} c_{j, \sigma}\right]+U \sum_{j}\left[c_{j, \uparrow}^{\dagger} c_{j, \uparrow}-1 / 2\right]\left[c_{j, \downarrow}^{\dagger} c_{j, \downarrow}-1 / 2\right]-\mu \sum_{\sigma} \hat{N}_{\sigma}-2 \mu_{0} H \hat{S}_{z}$,

where $c_{j, \sigma}^{\dagger}$ and $c_{j, \sigma}$ are the creation and annihilation operators, respectively, for electrons at the site $j$ with spin projection $\sigma=\uparrow, \downarrow$. In what follows $k_{F \sigma}=\pi n_{\sigma}$ and $k_{F}=\left[k_{F \uparrow}+k_{F \downarrow}\right] / 2=$ $\pi n / 2$, where $n_{\sigma}=N_{\sigma} / N_{a}$ and $n=N / N_{a}$, and $N_{\sigma}$ and $N_{a}$ are the number of $\sigma$ electrons and lattice sites, respectively $\left(N=\sum_{\sigma} N_{\sigma}\right)$. The results refer to all finite values of $U$, electron densities $0<n<1$, and spin densities $0<m<n$. This problem can be diagonalized using the BA [4]. This solution refers to a pseudoparticle operator basis, as was well established in Refs. [6.:7] (the pseudoparticle Hamiltonian parameters and phase shifts we refer below are studied in detail in these papers). At constant values of the electron numbers this description of the problem is very similar to Fermi-liquid theory, except for two main differences: (i) the $\uparrow$ and $\downarrow$ quasiparticles are replaced by the $c$ and $s$ pseudoparticles and (ii) the discrete pseudoparticle momentum (pseudomomentum) is of the usual form $q_{j}=\frac{2 \pi}{N_{a}} I_{j}^{\alpha}$ but the numbers $I_{j}^{\alpha}$ are not always integers. They are integers or half-integers depending whether the number of particles in the system is even or odd. This plays a central role in the present problem. The actual ground state of (1) is described in terms of the above $c$ and $s$ pseudoparticles which are created and annihilated by fermionic operators $b_{q, \alpha}^{\dagger}$ and $b_{q, \alpha}$, respectively (where $\alpha=c, s$ ). The ground state, $\left|0 ; N_{c}=N_{\uparrow}+N_{\downarrow}, N_{s}=N_{\downarrow}\right\rangle$, and low-energy Hamiltonian eigenstates can be completely described in terms of the occupations of these excitations. (Below we use often the alternative notation for the ground state, $\left|0 ; N_{\sigma}, N_{-\sigma}\right\rangle$.) The $c$ and $s$ pseudoparticles are non-interacting at the small-momentum and low-energy fixed 
point and the spectrum is described in terms of bands in a pseudo-Brillouin zone which goes between $q_{c}^{(-)} \approx-\pi$ and $q_{c}^{(+)} \approx \pi$ for the $c$ pseudoparticles and $q_{s}^{(-)} \approx-k_{F \uparrow}$ and $q_{s}^{(+)} \approx k_{F \uparrow}$ for the $s$ pseudoparticles. In the ground state these are occupied for $q_{F \alpha}^{(-)} \leq q \leq q_{F \alpha}^{(+)}$, where the pseudo-Fermi points are such that $q_{F c}^{( \pm)} \approx \pm 2 k_{F}$ and $q_{F s}^{( \pm)} \approx \pm k_{F \downarrow}$. Often, it is useful to introduce the quantum number $\iota= \pm 1$ which defines the right $(\iota=1)$ and left $(\iota=-1)$ pseudoparticles of momentum $\tilde{q}=q-q_{F \alpha}^{( \pm)}$. At higher energies and (or) large momenta the pseudoparticles start to interact (this is the price paid for the choice of a fermionic statistics!) via zero-momentum transfer forward-scattering processes. As in a Fermi liquid, these are associated with $f$ functions whose values at the pseudo-Fermi points define the Landau parameters, $F_{\alpha \alpha^{\prime}}^{j}=\frac{1}{2 \pi} \sum_{\iota= \pm 1}(\iota)^{j} f_{\alpha \alpha^{\prime}}\left(q_{F \alpha}^{( \pm)}, \iota q_{F \alpha^{\prime}}^{( \pm)}\right)$, where $j=0,1$. Their expressions involve the pseudoparticle group velocities $v_{\alpha}= \pm v_{\alpha}\left(q_{F \alpha}^{( \pm)}\right)$and the parameters $\xi_{\alpha \alpha^{\prime}}^{j}=\delta_{\alpha, \alpha^{\prime}}+\Phi_{\alpha \alpha^{\prime}}\left(q_{F \alpha}^{(+)}, q_{F \alpha^{\prime}}^{(+)}\right)+(-1)^{j} \Phi_{\alpha \alpha^{\prime}}\left(q_{F \alpha}^{(+)}, q_{F \alpha^{\prime}}^{(-)}\right)$, where $j=0,1$ and $\Phi_{\alpha \alpha^{\prime}}\left(q, q^{\prime}\right)$ is a two-pseudoparticle phase shift.

Our task is finding the relationship between the electronic operators $c_{k, \sigma}^{\dagger}$ in momentum space and the pseudoparticle operators $b_{q, \alpha}^{\dagger}$. In this Letter we solve the problem at the relative momenta of ground-state pairs differing in $N_{\sigma}$ by one. Notice that the electron excitation is not an eigenstate of the interacting problem and, therefore, when the electronic operator acts onto the ground state it produces a multiparticle process in terms of the pseudoparticles. The study of the above ground-state pairs of the interacting problem (1) reveals that their relative momentum equals precisely the $U=0$ Fermi points, $\pm k_{F \sigma}$. We consider the case when the electron has spin projection, say, $\uparrow$ and momentum $k_{F \uparrow}$ (the construction is the same for the case of spin projection $\downarrow$ ). We define the quasiparticle operator, $\tilde{c}_{k_{F \uparrow} \uparrow}^{\dagger}$, which creates one quasiparticle with spin projection $\uparrow$ and momentum $k_{F \uparrow}$ as,

$$
\tilde{c}_{k_{F \uparrow}, \uparrow}^{\dagger}\left|0 ; N_{c}=N_{\uparrow}+N_{\downarrow}, N_{s}=N_{\downarrow}\right\rangle=\left|0 ; N_{c}=N_{\uparrow}+1+N_{\downarrow}, N_{s}=N_{\downarrow}\right\rangle .
$$

The quasiparticle operator defines a one-to-one correspondence between the addition of one electron to the system and the creation of one quasiparticle, exactly as we expect from the 
Landau theory in 3D: the electronic excitation, $c_{k_{F \uparrow}, \uparrow}^{\dagger}\left|0 ; N_{c}=N_{\uparrow}+N_{\downarrow}, N_{s}=N_{\downarrow}\right\rangle$, defined at the Fermi momentum but arbitrary energy, contains a single quasiparticle, as we show below. We will study this excitation as we take the energy to be zero, where the problem is equivalent to Landau's. Since we are discussing the problem of addition or removal of one particle the boundary conditions play a crucial role. When we add or remove one electron from the many-body system we have to consider the transitions between states with integer and half-integer quantum numbers $I_{j}^{\alpha}$. The transition between two ground states differing in the number of electrons by one is then associated with two different processes: a backflow in the Hilbert space of the pseudoparticles with a shift of all the pseudomomenta by $\pm \frac{\pi}{N_{a}}$ and the creation of one or a pair of pseudoparticles at the pseudo-Fermi points. We find that the backflow is described in terms of a unitary operator,

$$
U_{\alpha}(\delta q)=\exp \left\{-\delta q \sum_{q^{\prime}}\left[\frac{\partial}{\partial q^{\prime}} b_{q^{\prime}, \alpha}^{\dagger}\right] b_{q^{\prime}, \alpha}\right\}
$$

From the expressions of the ground-state pseudoparticle generators the following relation between the quasiparticle and the pseudoparticles follows,

$$
\tilde{c}_{ \pm k_{F \uparrow} \uparrow}^{\dagger}=b_{q_{F c}^{( \pm)}, c}^{\dagger} U_{s}^{ \pm 1}, \quad \tilde{c}_{ \pm k_{F \downarrow} \downarrow}^{\dagger}=b_{q_{F c}^{( \pm)}, c}^{\dagger} b_{q_{F s}^{( \pm)}, s}^{\dagger} U_{c}^{ \pm 1}
$$

where $U_{\alpha}^{ \pm 1}=U_{\alpha}\left(\mp \frac{\pi}{N_{a}}\right)$. According to Eq. (4) the $\sigma$ quasiparticles are many-pseudoparticle objects which recombine the pseudoparticle colors $c$ and $s$ (charge and spin in the limit $\left.m=n_{\uparrow}-n_{\downarrow} \rightarrow 0[7]\right)$ giving rise to spin projection $\uparrow$ and $\downarrow$ and have Fermi surfaces at $\pm k_{F \sigma}$. However, note that two-quasiparticle objects can be of two-pseudoparticle character because the product of the two corresponding many-pseudoparticle operators is such that $U_{\alpha}^{+1} U_{\alpha}^{-1}=\mathbb{1}$, as for the triplet pair $\tilde{c}_{+k_{F \uparrow} \uparrow}^{\dagger} \tilde{c}_{-k_{F \uparrow}, \uparrow}^{\dagger}=b_{q_{F c}^{(+)}, c}^{\dagger} b_{q_{F c}^{(-)}, c}^{\dagger}$

In order to relate the quasiparticle operators $\tilde{c}_{ \pm k_{F \sigma}, \sigma}^{\dagger}$ to the electronic operators $c_{ \pm k_{F \sigma}, \sigma}^{\dagger}$ we have combined a generator pseudoparticle analysis and a suitable Lehmann representation with conformal-field theory [5,7]. We measure the energy $\omega$ from the initial chemical potential $\mu\left(N_{\sigma}, N_{-\sigma}\right)$ (ie, we consider $\left.\mu\left(N_{\sigma}, N_{-\sigma}\right)=0\right)$. As in a Fermi liquid, we find that the one-electron renormalization factor $Z_{\sigma}(\omega)$ has a crucial role in the above relation. This 
factor is given by the small- $\omega$ leading-order term of $\left|1-\frac{\partial \operatorname{Re} \Sigma_{\sigma}\left( \pm k_{F \sigma}, \omega\right)}{\partial \omega}\right|^{-1}$, where $\Sigma_{\sigma}(k, \omega)$ is the $\sigma$ self energy. Remarkably, we found the following low- $\omega$ expression, $\operatorname{Re} \Sigma_{\sigma}\left( \pm k_{F \sigma}, \omega\right)=$ $\omega\left[1-\frac{\omega^{-1-\varsigma_{\sigma}}}{a_{0}^{\sigma}+\sum_{j=1,2,3, \ldots} a_{j}^{\sigma} \omega^{4 j}}\right]$, where $a_{j}^{\sigma}$ are constants and $\varsigma_{\uparrow}=-2+\sum_{\alpha} \frac{1}{2}\left[\left(\xi_{\alpha c}^{1}-\xi_{\alpha s}^{1}\right)^{2}+\left(\xi_{\alpha c}^{0}\right)^{2}\right]$ and $\varsigma_{\downarrow}=-2+\sum_{\alpha} \frac{1}{2}\left[\left(\xi_{\alpha s}^{1}\right)^{2}+\left(\xi_{\alpha c}^{0}+\xi_{\alpha s}^{0}\right)^{2}\right]$ are $U, n$, and $m$ dependent exponents which for $U>0$ are negative and such that $-1<\varsigma_{\sigma}<-1 / 2$. Therefore, both the real part of the Green function $\operatorname{Re} G_{\sigma}\left( \pm k_{F \sigma}, \omega\right)$ and the lifetime $\tau_{\sigma}=1 / \operatorname{Im} \Sigma_{\sigma}\left( \pm k_{F \sigma}, \omega\right)$ diverge when $\omega \rightarrow 0$ as $\omega^{\varsigma_{\sigma}}$, yet $Z_{\sigma}(\omega)=\frac{a_{0}^{\sigma}}{\left|\varsigma_{\sigma}\right|} \omega^{1+\varsigma_{\sigma}}$ vanishes in that limit and there is no overlap between the quasiparticle and the electron, in contrast to a Fermi liquid. In the different three limits $U \rightarrow 0, m \rightarrow 0$, and $m \rightarrow n$ the exponents $\varsigma_{\uparrow}$ and $\varsigma_{\downarrow}$ are are equal and given by $-1,-2+\frac{1}{2}\left[\frac{\xi_{0}}{2}+\frac{1}{\xi_{0}}\right]^{2}$, and $-\frac{1}{2}-\eta_{0}\left[1-\frac{\eta_{0}}{2}\right]$, respectively. Here the $m \rightarrow 0$ parameter $\xi_{0}$ changes from $\xi_{0}=\sqrt{2}$ at $U=0$ to $\xi_{0}=1$ as $U \rightarrow \infty$ and $\eta_{0}=\left(\frac{2}{\pi}\right) \tan ^{-1}\left(\frac{4 t \sin (\pi n)}{U}\right)$.

Our method allows the identification of which particular Hamiltonian eigenstates contribute to the above $a_{j}^{\sigma} \omega^{4 j}$ corrections and leads to the following relation between the electron and quasiparticle operators

$$
c_{ \pm k_{F \sigma}, \sigma}^{\dagger}=\sqrt{Z_{\sigma}(\omega)\left|\varsigma_{\sigma}\right|}\left[1+\omega^{2} \sum_{\alpha, \alpha^{\prime}, \iota= \pm 1} C_{\alpha, \alpha^{\prime}}^{\iota} \hat{\rho}_{\alpha, \iota}\left(\iota \frac{2 \pi}{N_{a}}\right) \hat{\rho}_{\alpha^{\prime},-\iota}\left(-\iota \frac{2 \pi}{N_{a}}\right)+\mathcal{O}\left(\omega^{4}\right)\right] \tilde{c}_{ \pm k_{F \sigma}, \sigma}^{\dagger},
$$

where $C_{\alpha, \alpha^{\prime}}^{\iota}$ are constants and $\hat{\rho}_{\alpha, \iota}(k)=\sum_{\tilde{q}} b_{\tilde{q}+k, \alpha, \iota}^{\dagger} b_{\tilde{q}, \alpha, \iota}$ is a pseudoparticle-pseudohole operator. When $\omega \rightarrow 0$ this relation refers to a singular transformation because $Z_{\sigma}(\omega)$ vanishes in that limit. Combining Eqs. (4) and (5) gives the electron operator in the pseudoparticle basis. The singular nature of the transformation (5) which maps the 0renormalization-factor electron onto the 1-renormalization-factor quasiparticle explains the perturbative character of the pseudoparticle-operator basis [7]. It is this perturbative character that determines the form of expression (5) which except for the non-classical exponent in the $\sqrt{Z_{\sigma}(\omega)}=\sqrt{\frac{a_{0}^{\sigma}}{\left|\varsigma_{\sigma}\right|}} \omega^{\frac{1+\varsigma_{\sigma}}{2}}$ factor (absorbed by the electron-quasiparticle transformation) includes only classical exponents, as in a Fermi liquid. Combining the relation $\tilde{c}_{k_{F \sigma}, \sigma}^{\dagger}\left|0 ; N_{\sigma}, N_{-\sigma}\right\rangle=\left|0 ; N_{\sigma}+1, N_{-\sigma}\right\rangle$ with Eq. (5) we find that $\sqrt{Z_{\sigma}(\omega)\left|\varsigma_{\sigma}\right|}=$ $\left|\left\langle 0 ; N_{\sigma}+1, N_{-\sigma}\left|c_{k_{F \sigma}, \sigma}^{\dagger}\right| 0 ; N_{\sigma}, N_{-\sigma}\right\rangle\right| \propto \omega^{\frac{1+\varsigma_{\sigma}}{2}}$. In the present thermodynamic limit this result is only valid in the limit $\omega \rightarrow 0$ and confirms that this amplitude vanishes, the way it 
goes to zero with the excitation energy being relevant for comparison with other vanishing matrix elements: the higher-order contributions to expression (5) are associated with lowenergy excited Hamiltonian eigenstates orthogonal to the ground states and whose matrixelement amplitudes vanish as $\omega^{\frac{1+\varsigma \sigma+4 j}{2}}$ (with $2 j$ the number of pseudoparticle-pseudohole processes relative to $\left|0 ; N_{\sigma}+1, N_{-\sigma}\right\rangle$ and $\left.j=1,2,3, \ldots\right)$. Therefore, the leading-order term of (5) and the exponent $\varsigma_{\sigma}$ fully control the low-energy overlap between the $\pm k_{F \sigma}$ quasiparticles and electrons and determines the expressions of all $k= \pm k_{F \sigma}$ one-electron low-energy quantities. For instance, we find for the $\sigma$ spectral function $A_{\sigma}\left( \pm k_{F \sigma}, \omega\right) \propto \omega^{\varsigma_{\sigma}}$. (For a numerical study see Ref. [8].) Furthermore, $A_{\sigma}(k, \omega)$ (and $\operatorname{Re} G_{\sigma}(k, \omega)$ ) vanishes when $\omega \rightarrow 0$ for all momentum values except at the non-interacting Fermi-points $k= \pm k_{F \sigma}$. It follows that for $\omega \rightarrow 0$ the density of states, $D_{\sigma}(\omega)=\sum_{k} A_{\sigma}(k, \omega)$, results, exclusively, of contributions from the peaks centered at $k= \pm k_{F \sigma}$ and is such that $D_{\sigma}(\omega) \propto \omega A_{\sigma}\left( \pm k_{F \sigma}, \omega\right)$. It is known that $D_{\sigma}(\omega) \propto \omega^{\nu_{\sigma}}$, where $\nu_{\sigma}$ is the exponent of the equal-time momentum distribution expression, $N_{\sigma}(k) \propto\left|k \mp k_{F \sigma}\right|^{\nu_{\sigma}}$ 迎. We find the relation $\varsigma_{\sigma}=\nu_{\sigma}-1$, in agreement with the above analysis. However, this simple relation does not imply that the equal-time expressions provide full information on the small-energy instabilities. For instance, in addition to the momentum values $k= \pm k_{F \sigma}$ and in contrast to the spectral function, $N_{\sigma}(k)$ shows singularities at $k= \pm\left[k_{F \sigma}+2 k_{F-\sigma}\right]$ [5]. Therefore, only the direct low-energy study reveals all the true instabilities of the quantum liquid. (In some Luttinger liquids $N(k) \propto\left|k \mp k_{F}\right|^{\nu}$ with $\nu>1$ [11]. Then, $A\left( \pm k_{F}, \omega\right) \propto \omega^{\nu-1}$ does not diverge.) The electron - quasiparticle low-energy overlap also determines the behavior of the two-electron vertex function at the Fermi momenta and small energy. As usually [11, we choose the energy variables in such a way that the combinations $\omega_{1}+\omega_{2}, \omega_{1}-\omega_{3}$, and $\omega_{1}-\omega_{4}$ are all equal to $\omega$. We find that the vertex function diverges as $\Gamma_{\sigma \sigma^{\prime}}^{\iota}\left(k_{F \sigma}, \iota k_{F \sigma^{\prime}} ; \omega\right) \propto$ $\omega^{-2-\varsigma_{\sigma}-\varsigma_{\sigma^{\prime}}}$, where $\iota= \pm 1$. Further, we could evaluate the following closed-form expression $\Gamma_{\sigma \sigma^{\prime}}^{\iota}\left(k_{F \sigma}, \iota k_{F \sigma^{\prime}} ; \omega\right)=\frac{1}{\left|\varsigma_{\sigma} \varsigma_{\sigma^{\prime}}\right| Z_{\sigma}(\omega) Z_{\sigma^{\prime}}(\omega)}\left\{\sum_{\iota^{\prime}= \pm 1}\left(\iota^{\prime}\right)^{\frac{1-\iota}{2}}\left[v_{\rho}^{\iota^{\prime}}+\left(\delta_{\sigma, \sigma^{\prime}}-\delta_{\sigma,-\sigma^{\prime}}\right) v_{\sigma_{z}}^{\iota^{\prime}}\right]-\delta_{\sigma, \sigma^{\prime}} \tilde{v}_{\sigma}\right\}$, where $v_{\rho}^{\iota}$ and $v_{\sigma_{z}}^{\iota}$ are given in Table I and involve only the pseudoparticle velocities and Landau parameters referred above. (We have not derived the expression for the velocity $\tilde{v}_{\sigma}$. Note, 
however, that the relevant quantity for the low-energy physics is $\tilde{v}_{\sigma}$-independent and reads $\left.\delta_{\sigma, \sigma^{\prime}} \tilde{v}_{\sigma}+\left|\varsigma_{\sigma} \varsigma_{\sigma^{\prime}}\right| Z_{\sigma}(\omega) Z_{\sigma^{\prime}}(\omega) \Gamma_{\sigma \sigma^{\prime}}^{\iota}\left(k_{F \sigma}, \iota k_{F \sigma^{\prime}} ; \omega\right).\right)$

Let us now consider the excitation energies $\Delta E_{s p}=\omega_{\sigma,-\sigma}^{0}-\omega_{\sigma}^{0}-\omega_{-\sigma}^{0}$ and $\Delta E_{t p \sigma}=\omega_{\sigma, \sigma}^{0}-$ $2 \omega_{\sigma}^{0}$, where $\omega_{\sigma}^{0}=E_{0}\left(N_{\sigma}+1, N_{-\sigma}\right)-E_{0}\left(N_{\sigma}, N_{-\sigma}\right), \omega_{\sigma,-\sigma}^{0}=E_{0}\left(N_{\sigma}+1, N_{-\sigma}+1\right)-E_{0}\left(N_{\sigma}, N_{-\sigma}\right)$, and $\omega_{\sigma, \sigma}^{0}=E_{0}\left(N_{\sigma}+2, N_{-\sigma}\right)-E_{0}\left(N_{\sigma}, N_{-\sigma}\right)$ are ground-state excitation energies. We have evaluated the exact expressions of these vanishing energies which to first order in $\frac{1}{N_{a}} \mathrm{read}$, $\Delta E_{s p}=\frac{\pi}{N_{a}}\left[v_{c}+F_{c c}^{0}+F_{c s}^{0}-\left(v_{s}+F_{s s}^{1}\right)+F_{c s}^{1}\right], \Delta E_{t p \uparrow}=\frac{\pi}{N_{a}}\left[v_{c}+F_{c c}^{0}-\left(v_{c}+F_{c c}^{1}\right)-\left(v_{s}+F_{s s}^{1}\right)+2 F_{c s}^{1}\right]$, and $\Delta E_{t p \downarrow}=\frac{\pi}{N_{a}}\left[v_{c}+F_{c c}^{0}+v_{s}+F_{s s}^{0}+2 F_{c s}^{0}-\left(v_{s}+F_{s s}^{1}\right)\right]$. Again, these expressions involve exclusively the velocities and Landau parameters. Defining the hole concentration $\delta=1-n$, while the above ground-state triplet pairing energies are always positive, the singlet pairing energy $\Delta E_{s p}$ is also positive except in a small concentration domain, $0<\delta<\delta_{c}$, where it is negative. This domain is larger for zero magnetization $m=0$. In this case the $U$-dependent critical concentration $\delta_{c}$ vanishes both in the limits $U \rightarrow 0$ and $U \rightarrow \infty$ and is maximum for an intermediate but relative large value of $U$. As in a Fermi liquid, we find $\tilde{c}_{k_{F \sigma}, \sigma}^{\dagger} \tilde{c}_{k_{F-\sigma},-\sigma}^{\dagger}\left|0 ; N_{\sigma}, N_{-\sigma}\right\rangle=\left|0 ; N_{\sigma}+1, N_{-\sigma}+1\right\rangle$ and $\tilde{c}_{k_{F \sigma}, \sigma}^{\dagger} \tilde{c}_{-k_{F \sigma}, \sigma}^{\dagger}\left|0 ; N_{\sigma}, N_{-\sigma}\right\rangle=$ $\left|0 ; N_{\sigma}+2, N_{-\sigma}\right\rangle$. This implies that $\Delta E_{s p}$ and $\Delta E_{t p \sigma}$ are quasiparticle pairing energies. Therefore, for $0<\delta<\delta_{c}$ there is attraction between quasiparticles in the singlet Cooperpair channel. In a Fermi liquid this would imply a singlet-superconductivity instability for hole concentrations $0<\delta<\delta_{c}$. However, since in the limit of vanishing energy the electrons and quasiparticles have no overlap the quasiparticle attraction does not necessarily imply the occurrence of such superconductivity instability. We evaluated the corresponding response functions with the results $\operatorname{Re} \chi_{s c}\left( \pm 2 k_{F}, \omega\right)$ and $\operatorname{Im} \chi_{s c}\left( \pm 2 k_{F}, \omega\right) \propto \omega^{\varsigma_{s c}}$, and $\operatorname{Re} \chi_{t c \sigma}(0, \omega)$ and $\operatorname{Im} \chi_{t c \sigma}(0, \omega) \propto \omega^{\varsigma_{t p \sigma}}$, where for $U>0$ the exponents are positive and such that $0<\varsigma_{s c}<1$, $0<\varsigma_{t c \uparrow}<1$, and $0<\varsigma_{t c \downarrow}<2$. Therefore, in the 1D Hubbard model the low-energy electron - quasiparticle overlap is not strong enough for the quasiparticle - quasiparticle attraction in the singlet channel giving rise to a superconductivity instability.

One of the goals of this Letter was, in spite of the differences between the Luttingerliquid Hubbard chain and 3D Fermi liquids, detecting common features in these two limiting 
problems which we expect to be present in electronic quantum liquids in spatial dimensions $1<\mathrm{D}<3$. As in 3D Fermi liquids, we find that there are Fermi-surface quasiparticles in the Hubbard chain which connect ground states differing in the number of electrons by one and whose low-energy overlap with electrons determines the one-electron $\omega \rightarrow 0$ divergences. For instance, in spite of the vanishing electron density of states and renormalization factor, we find that the spectral function vanishes at all momenta values except at the Fermi surface where it diverges (as a Luttinger-liquid power law). While low-energy excitations are described by $c$ and $s$ pseudoparticle-pseudohole excitations which determine the $c$ and $s$ separation [7], the quasiparticles describe only ground-state - ground-state transitions and recombine $c$ and $s$ (charge and spin in the $m \rightarrow 0$ limit) giving rise to the spin projections $\sigma$ (see Eq. (4)). Importantly, we have written the electron operator at the Fermi surface in the pseudoparticle basis. The vanishing of the electron renormalization factor implies a singular character for the transformation (5) which leads to the above quasiparticles with renormalization factor 1 . Our exact results have confirmed that the electron renormalization factor can be related to a single matrix-element amplitude 9, 10]. Although in the Luttingerliquid case the form of the vertex function at the Fermi surface depends on the way that the energies go to zero [11], our study reveals that the Landau parameters which control the quasiparticle interaction energies $\Delta E_{s p}$ and $\Delta E_{t p \sigma}$ are determined by the finite renormalized vertex $\lim _{\omega \rightarrow 0}\left[Z_{\sigma}(\omega) Z_{\sigma^{\prime}}(\omega) \Gamma_{\sigma \sigma^{\prime}}^{\iota}\left(k_{F \sigma}, \iota k_{F \sigma^{\prime}} ; \omega\right)\right]$, as in a Fermi liquid. This justifies the finite forward-scattering functions $f_{\alpha \alpha^{\prime}}\left(q, q^{\prime}\right)$ and the perturbative character of the Hamiltonian (1) in the pseudoparticle basis [7]. Finally, from the existence of Fermi-surface quasiparticles both in the 1D and 3D limits, our results suggest their existence for quantum liquids in dimensions $1<\mathrm{D}<3$ and we predict the main role of increasing dimensionality being the strengthening of the electron - quasiparticle overlap 12. For instance, if this leads to a finite (yet small) vanishing-energy overlap, the pre-existing 1D singlet quasiparticle pairing induced by electronic correlations could lead to a real superconductivity instability.

We thank D. K. Campbell, F. Guinea, and K. Maki for illuminating discussions. This research was supported by the NSF under the Grant No. PHY89-04035. 


\section{REFERENCES}

$1 \quad$ F. D. M. Haldane, J. Phys. C 14, 2585 (1981).

2 J. Carmelo and A. A. Ovchinnikov, Cargèse lectures, unpublished (1990); J. Phys.: Condens. Matter 3, 757 (1991).

3 F. D. M. Haldane, Phys. Rev. Lett. 66, 1529 (1991); E. R. Mucciolo, B. Shastry, B. D. Simons, and B. L. Altshuler, Phys. Rev. B 49, 15197 (1994).

4 Elliott H. Lieb and F. Y. Wu, Phys. Rev. Lett. 20, 1445 (1968).

$5 \quad$ Holger Frahm and V. E. Korepin, Phys. Rev. B 42, 10553 (1990); ibid. 43, 5653 (1991); Masao Ogata, Tadao Sugiyama, and Hiroyuki Shiba, ibid. 43, 8401 (1991).

6 J. M. P. Carmelo, P. Horsch, and A. A. Ovchinnikov, Phys. Rev. B 45, 7899 (1992); ibid. 46, 14728 (1992).

7 J. M. P. Carmelo, A. H. Castro Neto, and D. K. Campbell, Phys. Rev. Lett. 73, 926 (1994); ibid., Phys. Rev. B 50, 3667 (1994); ibid. 3683 (1994); J. M. P. Carmelo and N. M. R. Peres, ibid. 51, 7481 (1995).

8 R. Preuss, A. Muramatsu, W. von der Linden, P. Dierterich, F. F. Assaad, and W. Hanke, Phys. Rev. Lett. 73, 732 (1994).

$9 \quad$ P. W. Anderson, Phys. Rev. Lett. 64, 1839 (1990).

10 Walter Metzner and Claudio Castellani, preprint (1994).

11 J. Sólyom, Adv. Phys. 28, 201 (1979); J. Voit, Phys. Rev. B 47, 6740 (1993); Walter Metzner and Carlo Di Castro, ibid. 16107 (1993).

12 J. M. P. Carmelo, F. Guinea, P. Horsch, and K. Maki, preprint (1995). 


\section{TABLE}

$\begin{array}{lll} & v_{\rho}^{\iota} & v_{\sigma_{z}}^{\iota} \\ \iota=-1 & v_{c}+F_{c c}^{1} & v_{c}+F_{c c}^{1}+4\left(v_{s}+F_{s s}^{1}-F_{c s}^{1}\right) \\ \iota=1 & \left(v_{s}+F_{s s}^{0}\right) / L^{0} & \left(v_{s}+F_{s s}^{0}+4\left[v_{c}+F_{c c}^{0}+F_{c s}^{0}\right]\right) / L^{0}\end{array}$

Table I - Expressions of the parameters $v_{\rho}^{\iota}$ and $v_{\sigma_{z}}^{\iota}$ in terms of the velocities $v_{\alpha}$ and Landau parameters $F_{\alpha \alpha^{\prime}}^{j}$, where $L^{0}=\left(v_{c}+F_{c c}^{0}\right)\left(v_{s}+F_{s s}^{0}\right)-\left(F_{c s}^{0}\right)^{2}$. 